\title{
Online Cooperative Feedback Control of Residential Community Microgrids with 100\% Renewable Energy
}

\author{
Kunyu Zuo, Lei Wu \\ Stevens Institute of Technology, Hoboken, NJ, USA \\ kzuo, lei.wu@stevens.edu
}

\begin{abstract}
The emerging of renewable distributed energy resources (DER) in the residential community opens the door to forming a residential community microgrid. However, traditional microgrid controls via the hierarchical feedforward tertiary, secondary, and primary control framework may not be effective for such residential community microgrids, because of high volatility, low inertia, and insufficiency of DERs. This paper discusses an online feedback scheme, which cooperates the three control layers in real time to improve operational stability of the microgrid. In addition, to economically dispatch scarce reserve, this paper deduces an increment cost model of battery storage assets based on their degradation costs and depth of discharges. The model is of low computational complexity, thus can be naturally embedded in the proposed online cooperative feedback control scheme to calculate marginal price in real-time. Small-signal analysis and Simulink simulation are conducted to illustrate the performances of the proposed online cooperative feedback control scheme.
\end{abstract}

\section{Introduction}

\subsection{Residential community microgrid}

In the smart grid era, renewable distributed energy resources (DER) emerged on the consumer side present a great potential in regulating local electric energy consumptions. As a matter of fact, it is promising to leverage these DERs and form an islanded residential community microgrid during emerging situations. This could improve energy resilience, when the main grid outage occurs during natural or man-made disasters.

Unlike bulk systems or sophisticated microgrids equipped with sufficient resources to adequately supply local loads in the islanded mode [1]-[2], a residential community microgrid with low inertial (i.e., lack of synchronous generators) may lead to large frequency and voltage deviations. In addition, the limited DERs in residential community microgrid would be operated out of safe states more frequently in order to meet microgrid regulations [3]-[4]. To this end, a more responsive and interactive control scheme - online cooperative feedback control, is needed to meet practical needs.

\subsection{Online cooperative feedback control}

In existing hierarchical control scheme designs for sophisticated microgrids [5], the three control layers are connected rather loosely. As shown in Figure 1, the primary, secondary, and tertiary control are activated at different time scales in a feedforward loop. Specifically, tertiary control is activated every 5 minutes to provide economic operating points of DERs; secondary control is triggered every 2-6 seconds to regulate frequency/voltage back to the nominal values; and primary control acts promptly against supply-demand imbalance [6].

However, the hierarchical feedforward control scheme as shown in Figure 1 may not guarantee operational stability of the renewable DER dominated residential community microgrid, because it cannot provide timely and effective feedback control actions to promptly adjust DER outputs against real-time variabilities. Moreover, even a relatively small disturbance or measurement error could easily induce noticeable steady-state errors in such a small system, leading to suboptimality [7].

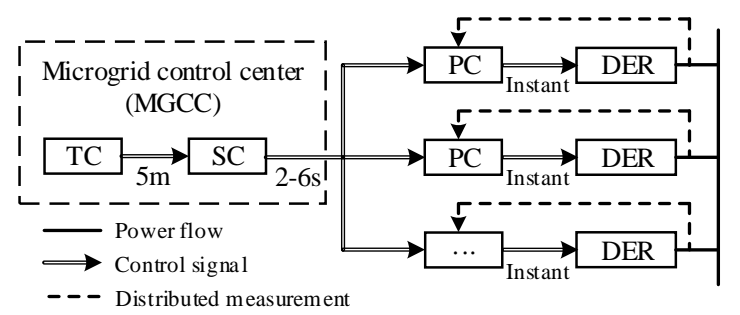

Figure 1. Hierarchical feedforward control of microgrid in islanded mode (PC: Primary Control, SC: Secondary Control, TC: Tertiary Control)

In order to improve the respond speed and optimality, reference [8] adopted a partial primal-dual gradient method to solve the tertiary control problem, 
and embedded each step of the iterative procedure into the secondary control, forming a real-time secondarytertiary control scheme. Similarly, reference [9] adopted the alternative direction method of multipliers (ADMM) approach to decompose the AC optimal power flow problem, and integrated each step of the adjustments into the primary control. Moreover, references [10]-[11] realized the real-time reception and execution of control signals by promptly changing frequency and power reference of the primary control.

It is clearly shown in references [8]-[11] that, compared with the hierarchical feedforward control, the hierarchical feedback control can better coordinate control actions of the three layers based on real-time information. That is, it could adjust outputs of DERs more rapid and accurately, enabling better stability and economic efficiency of the entire system. However, existing researches present and validate such a feedback coordination for only two control layers (i.e., primarysecondary coordination or primary-tertiary coordination), lacking a comprehensive understanding on systematic performance of the cooperative feedback control among all three layers. In this paper, we aim at establishing an online cooperative feedback control scheme, which covers all three layers to realize real-time interactions and adjustments of the DERs in the residential community microgrid in islanded mode, as shown in Figure 2.

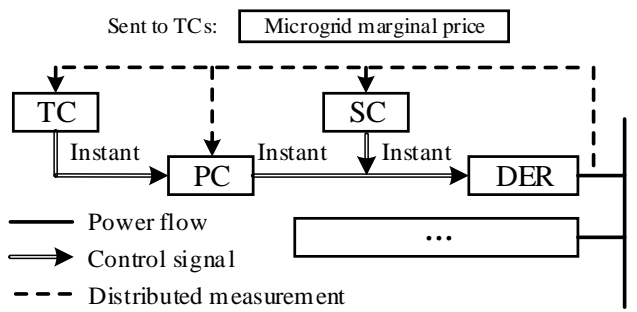

Figure 2. Online cooperative feedback control of microgrid in islanded mode

\subsection{Marginal cost model of battery storage to enable price-driven tertiary control}

A comprehensively designed microgrid is usually equipped with heterogeneous resources to adequately supply all local (critical) loads [1]-[2] in islanded mode. However, for a residential community microgrid with $100 \%$ renewable DERs and limited battery storage assets, it is challenging to maintain real-time supplydemand balance. The reasons are: (i) Supply is limited and volatile; and (ii) It is hard to categorize priorities of demands (i.e., it may be possible to categorize priorities of loads within each resident, however, it is hard to justify a resident's load is more critical than another).

Indeed, under the normal situation, the main grid supplies majority of electric energy needs of the community, while DERs are used to supplement the main grid to achieve economic and/or environmental benefits. To this end, when the main grid is down, the residential community may not have enough DER capacities to supply all residential loads in islanded mode. Nevertheless, certain residents may be willing to pay higher prices in return for electricity. Thus, it is practically viable to dispatch scarce resources through a price-driven tertiary control scheme for the residential community microgrid in islanded mode.

The price-driven tertiary control adopts demand curve and supply curve to dispatch scarce resources. Demand curve can be formulated by means of the price elasticity of demand [12]. Reference [13] modelled the supply curve via quadratic cost functions of fossil-fuel generators. In comparison, in the residential community microgrid dominated by renewable DERs, battery storage assets would be the only source that can set the supply curve. Levelized cost of energy (LCOE) is a general approach to model the cost of a battery storage, by averaging the life cycle costs (including capital cost, operation cost, and maintenance cost) into each hour [14]. However, it is a constant value that cannot accurately reflect the energy scarcity situations at different time periods (with respect to the availability and fluctuation of renewable DERs) and the dynamic degradation process of a battery storage through multiple charging-discharging cycles.

To this end, a degradation cost model of battery storage assets was studied in [15] to quantify the cost of each charge-discharge cycle according to its impacts on the life cycle costs. This model, however, is only suitable for quantifying battery storage cost during a certain time period via a centralized optimization framework, but cannot offer incremental cost of batteries for real-time price-driven tertial control. In this paper, we will refine and extend this model to derive an incremental cost model of batteries, and derive a supply curve to price electricity in the tertiary control of the residential community grid in islanded mode.

\subsection{Contributions}

This paper discusses an online cooperative feedback control scheme to improve operational stability and economics of residential community microgrid in islanded mode. Specifically, the proposed feedback control scheme is constructed by coordinating three controllers in real time via a feedback loop, as shown in Figure 2. The proposed control scheme includes a novel price-driven tertiary control to ensure economic performance of all DERs, a secondary control to maintain nominal frequency and voltage values, and a primary control to achieve supply-demand balance. 
Besides, tertiary control interacts with primary control in real time by providing updates on active/reactive power set points; Meanwhile, secondary control cooperates with primary control in real time to drive frequency/voltage back to reference points. Therefore, each controller presents a feedback structure and is also embedded in the entire feedback control scheme, guaranteeing their individual and collaborative performance to pursue operational stability and economics of the residential community microgrid.

Moreover, the small-signal analysis [16] and the Simulink Simscape Electrical based simulation [17] are conducted to verify and demonstrate the stability and economic efficiency of the proposed online cooperative feedback control scheme. Specifically, the small-signal analysis determines whether the system converges by calculating eigenvalues of system characteristic equations, and the Simscape Electrical platform further simulates the dynamic performance of the proposed online cooperative feedback control scheme via a smallscale residential community microgrid setup.

The main contributions of the paper include:

1) An online cooperative feedback control scheme is proposed to enhance operational stability and economics of the residential community microgrid in islanded mode, which is dominated by renewable DERs and limited battery storage assets;

2) The proposed incremental cost model of batteries is used to enable the automatic price response of DERs and loads through a price-driven tertiary control, achieving the decentralized economic operation of residential community microgrid in islanded mode;

3) Small-signal analysis and control loop simulation are conducted to verify operational stability and economic efficiency of the proposed online cooperative feedback control scheme.

The rest of the paper is organized as follows. Section 2 describes the three control layers in detail. Section 3 presents the online cooperative feedback control structure and the small-signal stability analysis. Simscape Electrical based simulation for the community microgrid in islanded mode is conducted in Section 4. The paper is concluded in Section 5.

\section{Three control layers of microgrid}

A microgrid in islanded mode is operated via a three-layer hierarchical control scheme. Primary control sustains the instantaneous supply-demand balance. It, however, induces deviations on microgrid frequency and voltage. Consequently, secondary control regulates frequency and voltage back to the reference values. Moreover, tertiary control is responsible for operating the overall microgrid in an economic manner.

\subsection{Primary control}

In the traditional primary control, when the supplydemand imbalance occurs, the rotation speed of rotors in synchronous generators will be altered with respect to their inertia. The altered rotation speed changes the system frequency, which drives synchronous generators, in coordination with other frequencyresponsive devices, to reinstall power balance. However, a residential community microgrid dominated by renewable DERs is lack of power inertia, which potentially induces poor transient stability performance. To this end, a common technique is to apply the droop control on voltage sources (e.g., chemical battery) and controllable inverters to imitate the frequency change of synchronous generators and coordinate their responses to mitigate supply-demand imbalance.

Three types of primary droop control strategies can be designed corresponding to distinct $\mathrm{R} / \mathrm{X}$ ratios of distribution lines: (i) $\mathrm{R} \ll \mathrm{X}$; (ii) $\mathrm{R} \gg \mathrm{X}$; and (iii) $\mathrm{R} \approx \mathrm{X}$. Under different situations, real and reactive power flows are dominated by distinct driven factors. To this end, three droop control strategies can be designed against distinct $\mathrm{R} / \mathrm{X}$ ratios. Specifically, with $\mathrm{R} \ll \mathrm{X}$, the primary control can be achieved by $P-f$ droop, while $P-V$ droop and virtual frame transformation droop are respectively used in the second and the third situations.

We take the first situation for the detailed explanation. With $\mathrm{R} \ll \mathrm{X}$, active power flow $P_{i j}$ on branch $i j$ can be calculated as in (1) [18]. $X_{i j}$ is impedance of branch $i j ; \theta_{i}$ and $V_{i}>0$ are phasor angle and voltage magnitude of node $i$; and $\mathcal{V}$ is the set of nodes in a microgrid.

$P_{i j}=V_{i} V_{j} \sin \left(\theta_{i}-\theta_{j}\right) / X_{i j} ; \quad i, j \in \mathcal{V}$ (1)

As $V_{i}$ and $V_{j}$ are close to the per unit value under normal operation, with fixed $X_{i j}$, active power flow $P_{i j}$ is dominated by the phase angle difference $\left(\theta_{i}-\theta_{j}\right)$. To this end, active power injection $P_{i}$ of DERs at node $i$ can be controlled by changing $\theta_{i}$ with reference to $\theta_{j}$. This is realized via the active power droop control, which changes $\dot{\theta}_{i}$ (i.e. $f_{i}^{*}-f_{i}$ ) according to the difference between measured operating status $P_{i}$ and set point $P_{i}^{*}$ (i.e., $P_{i}^{*}-P_{i}$ ), as shown in (2). $m_{i}<0$ is the droop coefficient; $f_{i}^{*}$ is the nominal frequency; $\mathcal{V}_{C}$ denotes the set of controllable batteries equipped with primary controllers. Hereby, the negative feedback loop between (1) and (2) can in principal achieve convergence [19]. $\Delta f_{i}=f_{i}^{*}-f_{i}=m_{i}\left(P_{i}^{*}-P_{i}\right) ; \quad i \in \mathcal{V}_{C}(2)$

Moreover, according to the power sharing rule (3) among multiple DERs under the droop control, the unified frequency deviation $\Delta f_{\text {sync }}$ after convergence can be calculated as in (4).

$\Delta f_{\text {sync }}=m_{i} \Delta P_{i}=m_{j} \Delta P_{j} ; \quad i \in \mathcal{V}_{C}$ 
$\Delta f_{\text {sync }}=\sum_{i \in \mathcal{V}_{C}} P_{i} / \sum_{i \in \mathcal{V}_{C}}\left(1 / m_{i}\right)$

In terms of reactive power droop control, with $\mathrm{R} \ll \mathrm{X}$, reactive power flow $Q_{i j}$ on branch $i j$ can be calculated as in (5). Similarly, assuming $\cos \left(\theta_{i}-\theta_{j}\right) \approx$ 1 , reactive power flow $Q_{i j}$ is dominated by the voltage difference $V_{i j}=V_{i}-V_{j}$ [18]. That is, adjusting $V_{i}$ with reference to $V_{j}$ can control reactive power injection $Q_{i}$ at node $i$. Thus, the control signal is given as in (6).

$Q_{i j}=V_{i}\left[V_{i}-V_{j} \cos \left(\theta_{i}-\theta_{j}\right)\right] / X_{i j} ; \quad i, j \in \mathcal{V}_{C}$ (5) $\Delta V_{i}=V_{i}^{*}-V_{i}=n_{i}\left(Q_{i}^{*}-Q_{i}\right) ; \quad i \in \mathcal{V}_{C}$ (6)

It is noteworthy that active droop control acts on frequency $\dot{\theta}_{i}$ instead of phasor angle $\theta_{i}$. The reasons are: (1) frequency is a globe signal that can effectively coordinate droop control actions of all DERs, and (ii) modifying $\dot{\theta}_{i}$ can yield smoother nodal profiles than modifying phasor angle $\theta_{i}$ in a sine system. Motivated by this, [20] adopts $\dot{V}_{i}$ instead of $V_{i}$ for reactive power droop control. However, it does not present similar advantages as using $\dot{\theta}_{i}$ for active power droop control. One major reason is that $\dot{V}_{i}$ varies over nodes, and individual DER controllers cannot simply set their voltage targets to a uniformed value as global frequency. Therefore, $V_{i}$ droop for reactive power (6), as a direct and effective control method, remains the main trend.

\subsection{Secondary control}

As the droop-based primary control induces deviations on frequency and voltage against their nominal values, the secondary control is further used to regulate such deviations.

Frequency regulation is realized via a proportionalintegral (PI) controller [3] using frequency deviation as the input, as shown in (7). $k_{f, P}^{\mathrm{II}}$ and $k_{f, I}^{\mathrm{II}}$ denote proportional and integral coefficients. To this end, $\Delta f_{i}^{\mathrm{II}}$, the regulation command calculated by the secondary control, is sent to the governor to continuously modify the frequency reference $f_{i}^{*}$. Consequently, it gradually adjusts outputs of DERs to restore the frequency.

$\Delta f_{i}^{\mathrm{II}}=k_{f, P}^{\mathrm{II}} \Delta f_{i}+k_{f, I}^{\mathrm{II}} \int \Delta f_{i}(t) d t ; \quad i \in \mathcal{V}_{C}$ (7)

Voltage regulation can be similarly implemented as in (8), where $\Delta V_{i}=V_{i}^{*}-V_{i}$, and $k_{v, P}^{\mathrm{II}}$ and $k_{v, I}^{\mathrm{II}}$ are the proportional and integral coefficients. The secondary control signal $\Delta V_{i}^{\mathrm{II}}$, in a feedback fashion, is continuously added on the voltage reference $V_{i}^{*}$, which gradually drives $V_{i}$ back to the nominal voltage value. $\Delta V_{i}^{\mathrm{II}}=k_{v, P}^{\mathrm{II}} \Delta V_{i}+k_{v, I}^{\mathrm{II}} \int \Delta V_{i}(t) d t ; \quad i \in \mathcal{V}_{C}$ (8)

\subsection{Tertiary control}

Tertiary control aims at optimally dispatching flexible loads and batteries in the residential community microgrid to purse the economic operation.

The demand response characteristics of flexible loads are formulated via the price elasticity of demands [12] as in (9). $\varepsilon$ describes price elasticity of demand; $\rho_{L}$ and $\rho_{L}^{*}$ are the marginal price and normal electricity price of demand; $P_{L}$ and $P_{L}^{*}$ are actual dispatch level and normal load level of demand.

$\rho_{L}=\rho_{L}^{*}\left(P_{L} / P_{L}^{*}\right)^{-\varepsilon}$;

In this paper, we use a degradation cost model to calculate the marginal cost of batteries. The battery degradation cost is related to charging and discharging actions, and can be modelled via the depth of discharge (DOD) [21] as in (10)-(12). A larger DOD would accelerate battery aging, thus corresponding to a higher degradation cost.

$n_{L C}\left(d_{B}\right)=a *\left(d_{B}\right)^{-b}$;

$y\left(d_{B}\right)=n_{L C}\left(d_{B}\right) / n_{Y C}$

$C_{B}\left(d_{B}\right)=\frac{r(1+r)^{y\left(d_{B}\right)}}{(1+r)^{y\left(d_{B}\right)}-1} \frac{C_{\text {capital }}}{n_{Y C}}$

Equation (10) calculates the lifetime cycles $n_{L C}$ of a battery, where $d_{\mathrm{B}}$ is the DOD of the battery, $a$ and $b$ are battery specific paraments. Parameter $a$ is related to capital cost of a battery, and $b$ is usually set in the range of 1.9 to 2.1 [21]-[22]. With this, the lifetime $y$ of a battery can be calculated by the lifetime cycles divided by the annual cycles $n_{Y C}$ as in (11). Finally, the capital cost of the battery $C_{\text {capital }}$ can be spread out over the lifetime with a given discount rate $r$, deriving the cost $C_{B}$ per discharge cycle. With the setting of $b=2$ and $r=0,(10)-(12)$ can be equivalently converted into (13). $C_{B}\left(d_{B}\right)=d_{B}{ }^{2} C_{\text {capital }} / a$;

The relationship between net power dispatch $P_{B}$ (i.e., discharging minus charging) of the battery and the average DOD over a short time period of $\Delta t$ is formulated as in (14). $S_{B}$ and $E_{B}$ are the initial state of charge (SOC) and the energy of the battery for time interval $\Delta t$. Thus, the initial DOD is $1-S_{B}$, and the terminal DOD is $1-S_{B}+\eta P_{B} \Delta t / E_{B}$. With this, the average DOD during interval $\Delta t$ is calculated as the mean of initial and terminal DODs as in (14), where $\eta_{c h a}$ and $\eta_{d i s}$ are charging and discharging efficiencies. $d_{B}=1-S_{B}+\eta P_{B} \Delta t /\left(2 E_{B}\right) ; \quad \begin{array}{ll}\eta=1 / \eta_{\text {dis }} & P_{B} \geq 0 ; \\ \eta=\eta_{\text {cha }} & P_{B}<0 ;\end{array}$

Consequently, combining (13) and (14), the incremental cost for charging/discharging (a negative/ positive value) can be calculated as in (15), where $A=$ $C_{\text {capital }}(\eta \Delta t)^{2} /\left(2 a E_{B}{ }^{2}\right)$ and $B=C_{\text {capital }} \eta \Delta t(1-$ $\left.S_{B}\right) /\left(a E_{B}\right)$.

$\rho_{B}\left(P_{B}\right)=\frac{\partial C_{B}\left(P_{B}\right)}{\partial P_{B}}=A \cdot P_{B}+B$

In islanded mode, considering limited on-site DERs, the residential community microgrid is operated at a relatively low load level. Thus, network congestion may not be a major concern. In addition, voltage and frequency values are regulated by the secondary control 
to meet their corresponding limits. Moreover, ramping limits of DERs can be reflected via the time constants of the control loops. Thus, the optimal solution of the tertiary control for minimizing the total operation cost can be described by the equilibrium point as in (16), where $\rho_{M}$ is the marginal price of the microgrid. It describes that all nodes equilibrate at the same marginal price with the supply-demand balance.

$$
\rho_{L}=\rho_{B}\left(P_{B}\right)=\rho_{M} ; \quad \sum P_{i}=0 ; \quad i \in \mathcal{V} \text { (16) }
$$

The equilibrium point as described in (16) is achieved via the interaction between a decentralized tertiary control (17) of individual DERs and the centralized microgrid clearing to calculate $\rho_{M}$ (18). The tertiary control of DERs is designed as in (17), which is a feedback control to drive flexible loads (9) and batteries (15) towards the microgrid marginal price $\rho_{M}$. $\Delta P_{i}^{\mathrm{III}}$ is adjustment on power reference of dispatchable resource $i, k_{f, P}^{\mathrm{III}}$ and $k_{f, I}^{\mathrm{III}}$ denote the proportional and integral coefficients of the tertiary control. Equation (18) continuously calculates the microgrid marginal price $\rho_{M}$ as the mean value of marginal prices for all nodes. It offers the price guidance for the DERs to converge towards the optimal point as described in (16). $\Delta P_{i}^{\mathrm{III}}=k_{f, P}^{\mathrm{III}}\left(\rho_{i}-\rho_{M}\right)+k_{f, I}^{\mathrm{III}} \int\left(\rho_{i}-\rho_{M}\right) d t ;$

$\rho_{M}=\operatorname{mean}\left(\rho_{i}\right)$;

$i \in \mathcal{V}(18)$

\section{Online cooperative feedback control 3.1. Online cooperative feedback control design}

The hierarchical feedforward control scheme is widely adopted in power system controls, which executes the three control layers in a queue at different time scales, as shown in Figure 1. One major disadvantage of such a feedforward control scheme is that the three control layers are loosely connected, which may not achieve real-time operational stability and economics for the residential community microgrid.

To this end, this paper proposes an online cooperative feedback control design for residential community microgrid, so that each layer can tightly interact with others in real time. Specifically, (i) each of the three control layers is constructed via a feedback structure and executed in real time, as discussed in Section 2; and (ii) the three control layers are tightly connected and closely interacting with each other in real-time scale, as shown in Figure 2.

Figure 3 shows the block diagram of the proposed online cooperative feedback control. The explanations on symbols in Figure 3 can be referred to from equations (19) and (22). We take the active power control loop (as shown in the shaded area of Figure 3) for the detailed discussion. With an active power disturbance $\Delta P_{D}$, the voltage source node will immediately adjust its output $P_{B}$ to keep supply-demand balance, and its primary control will actively droop the output frequency of this voltage source node. Thus, the difference between the modified frequency and the system frequency reference gradually drives the phase angle difference between the voltage source node and the rest of the system. As the phase difference emerges, part of the active power compensated by this voltage source will be taken over by voltage sources on other nodes, triggering power flow redistribution of the microgrid as shown in (1).

However, although the primary control can reach supply-demand balance by activating power sharing among all nodes with respect to their droop coefficients, the frequency value at consensus would differ from the nominal value. Thus, the secondary control loop (7) is further applied on batteries in real-time, to continuously mitigate frequency deviation and pull the frequency back to the nominal value.

Moreover, the proposed tertiary control (17) is carried out according to the difference between the incremental cost of batteries (15) and microgrid marginal price (18). It generates control signals to adjust the power reference and interact with the primary control, until all DERs arrive the optimal point (16).

Hereby, the three control layers are tightly integrated in feedback loops to form the proposed online cooperative feedback control for the residential community microgrid, which can effectively sustain supply-demand balance, regulate voltage/frequency, and track the economic operation point.

\subsection{Small-signal stability analysis}

Small-signal analysis is used to analyze stability of the active and reactive power control loops in Figure 3. We establish the small-signal state space model of each loop, and calculate eigenvalues of the characteristic equation. Based on positions of eigenvalues, the stability and transient performance of the control loops can be theoretically analyzed.

For the active power control loop of the proposed online cooperative feedback control scheme, the entire procedure from active power disturbance $\Delta P_{D}(s)$ to active power adjustment $\Delta P_{B}(s)$ of DERs includes a set of processes in the state space, as shown in (19). The power flow gain (19a) is linearized according to (1).

Power flow gain: $G_{f}^{\mathrm{PF}}=V_{i} V_{j} \cos \left(\theta_{i}-\theta_{j}\right) / X_{i j}$

Primary controller: $G_{f}^{\mathrm{I}}=K_{f, P}^{\mathrm{I}} G_{f, F}^{\mathrm{I}}$

Primary control filter: $G_{f, F}^{\mathrm{I}}=1 /\left(1+\tau^{\mathrm{I}} s\right)$

Secondary controller: $G_{f}^{\mathrm{II}}=\left(K_{f, P}^{\mathrm{II}}+K_{f, I}^{\mathrm{II}} / s\right) G_{f, F}^{\mathrm{II}}$

Secondary control filter: $G_{f, F}^{\mathrm{II}}=1 /\left(1+\tau^{\mathrm{II}} s\right)$

Tertiary controller: $G_{f}^{\mathrm{III}}=A\left(K_{f, P}^{\mathrm{III}}+K_{f, I}^{\mathrm{II}} / s\right) G_{f, F}^{\mathrm{III}}$

Tertiary control filter: $G_{f, F}^{\mathrm{III}}=1 /\left(1+\tau^{\mathrm{III}} s\right)$

Frequency to theta controller: $G_{f}^{\mathrm{FT}}=2 \pi / s$ 


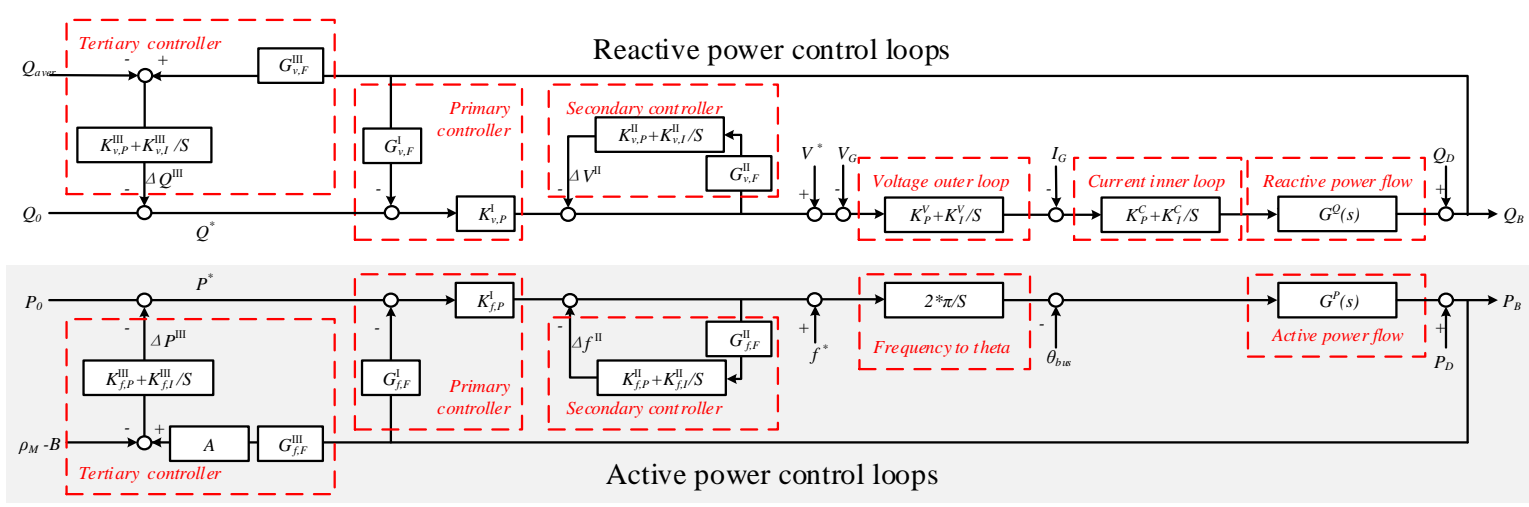

Figure 3. Block diagram of the proposed online cooperative feedback control

According to the Mason's gain formula, the transfer function of $\Delta P_{B}(s)$ over $\Delta P_{D}(s)$ can be expressed as in (20). With this, the active power characteristic equation $\Delta_{A P}$ is presented as in (21).

$\frac{\Delta P_{B}(s)}{\Delta P_{D}(s)}=1 /\left(1+G_{f}^{\mathrm{I}} G_{f}^{\mathrm{FT}} G_{f}^{\mathrm{PF}}+G_{f}^{\mathrm{II}}+G_{f}^{\mathrm{III}} K_{f, P}^{\mathrm{I}} G_{f}^{\mathrm{FT}} G_{f}^{\mathrm{PF}}\right)$

$\Delta_{A P}=\left(1+G_{f}^{\mathrm{I}} G_{f}^{\mathrm{FT}} G_{f}^{\mathrm{PF}}+G_{f}^{\mathrm{II}}+G_{f}^{\mathrm{III}} K_{f, P}^{\mathrm{I}} G_{f}^{\mathrm{FT}} G_{f}^{\mathrm{PF}}\right)$

Similarly, $\Delta Q_{D}(s)$ transfers to $\Delta Q_{B}(s)$ through a chain of processes as describes in (22). The voltage outer loop (22h) and the current inner loop (22i) regulate the nodal voltage and current. Consequently, the transfer function of $\Delta Q_{B}(s)$ over $\Delta Q_{D}(s)$ is shown as in (23), and the reactive power characteristic equation $\Delta_{R P}$ is given as in (24).

Power flow gain: $G_{v}^{\mathrm{PF}}=2 V_{i 0}-V_{j} \cos \left(\theta_{i}-\theta_{j}\right) / X_{i j}$ (22a)

Primary controller: $G_{v}^{\mathrm{I}}=K_{v, P}^{\mathrm{I}} G_{v, F}^{\mathrm{I}}$

Primary control filter: $G_{v, F}^{\mathrm{I}}=1 /\left(1+\tau^{\mathrm{I}} s\right)$

Secondary controller: $G_{v}^{\mathrm{II}}=\left(K_{v, P}^{\mathrm{II}}+K_{v, I}^{\mathrm{II}} / s\right) G_{v, F}^{\mathrm{II}}$

Secondary control filter: $G_{v, F}^{\mathrm{II}}=1 /\left(1+\tau^{\mathrm{II}} s\right)$

Tertiary controller: $G_{v}^{\mathrm{III}}=\left(K_{v, P}^{\mathrm{III}}+K_{v, I}^{\mathrm{III}} / s\right)$

Tertiary control filter: $G_{v, F}^{\mathrm{III}}=1 /\left(1+\tau^{\mathrm{III}} s\right)$

Voltage outer loop: $G_{V}=\left(K_{P}^{\mathrm{V}}+K_{I}^{\mathrm{V}} / s\right)$

Current inner loop: $G_{C}=\left(K_{P}^{\mathrm{C}}+K_{I}^{\mathrm{C}} / s\right)$

$\frac{\Delta Q_{B}(s)}{\Delta Q_{D}(s)}=1 /\left(1+G_{v}^{\mathrm{I}} G_{V} G_{I} G_{v}^{\mathrm{PF}}+G_{v}^{\mathrm{II}}+G_{v}^{\mathrm{III}} K_{v, P}^{I} G_{V} G_{I} G_{v}^{\mathrm{PF}}\right)$

$\Delta_{R P}=1+G_{v}^{\mathrm{I}} G_{V} G_{I} G_{v}^{\mathrm{PF}}+G_{v}^{\mathrm{II}}+G_{v}^{\mathrm{III}} K_{v, P}^{I} G_{V} G_{I} G_{v}^{\mathrm{PF}}$

The control parameters adopted in case studies are listed in the Appendix. By calculating roots of the characteristic equation (21), the five eigenvalues of the active power control loop are -98.7398, -59.3306, $3.9627,-1.8866$, and -0.0803 . All eigenvalues are located on the left-half plane (LHP), indicating that the active power control loop is stable and with good convergence performance.

Different from the active power control loop, the reactive power control loop may be vulnerable in stability, because nodal voltages are local signals and harder to coordinate. Root locus of the reactive power control loop is plotted in Figure 4. Since the poles/zeros near the origin dominate the system convergence performance, the blue block in Figure 4 is zoomed in to have a closer look. The green and red loci intersect with the imaginary axis at the gain of 16.8 , which is the critical gain value for ensuring stability. In our simulations, the power flow gain $G_{v}^{\mathrm{PF}}$ is set as 1 . Although the linearized power flow calcuation may introduce certain errors, the actual gain shall remain close to 1 and far smaller than 16.8. Thus, the reactive power control loop shall remain stable, which is also clearly verified via Simulink dynamic simulation in the next section. Nevertheless, there is a pair of complex roots, $(-1.27,1.39 \mathrm{i})$ and $(-1.27,-1.39 \mathrm{i})$, with the gain of 1 . Thus, the reactive power control loop will show underdamped oscillation against disturbance before eventually converging to a stable status.

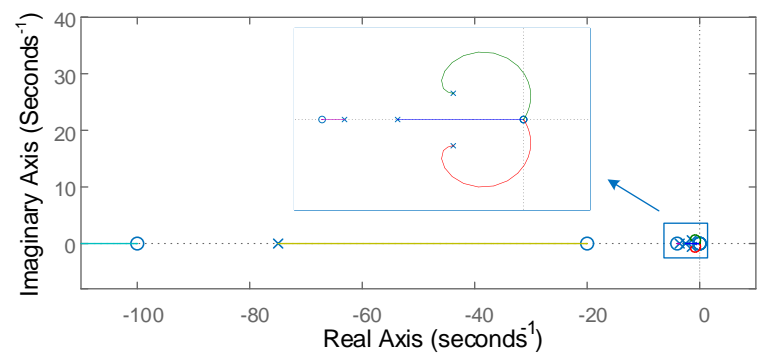

Figure 4. Root locus of the reactive power control loop

\section{Simulation}

A single-phase residential community microgrid with 5 nodes, as shown in Figure 5, is used to verify effectiveness of our proposed online cooperative feedback control scheme. The point of common coupling (PCC) is set as $240 \mathrm{~V}$. The base value of $3.5 \mathrm{kVA}$ is used for per-unit conversion. Solar PV1 is connected at node 3 via a grid-following inverter. PV1 is operated under the maximum power point tracking (MPPT) mode, and its power output depends on the realtime solar irradiation and temperature. Two batteries, BS1 and BS2, are connected at nodes 1 and 2 via gridforming inverters. Two frequency sensitive loads, L1 
and L2, are connected at nodes 4 and 5, and their pricesensitive demand response characters are modelled as in (8). Table 1 lists the control functionalities that individual assets offer in the simulations.

Table 1. DER functionalities in simulation

\begin{tabular}{|c|c|c|c|c|c|}
\hline & BS1 & BS2 & PV1 & L1 & $\mathrm{L} 2$ \\
\hline Primary control & $\sqrt{ }$ & $\sqrt{ }$ & $x$ & $\times$ & $\times$ \\
\hline Frequency response & $\sqrt{ }$ & $\sqrt{ }$ & $x$ & $\sqrt{ }$ & $\sqrt{ }$ \\
\hline Secondary control & o & $x$ & $x$ & $x$ & $x$ \\
\hline Feedforward TC & $\mathrm{o}$ & 0 & $x$ & o & o \\
\hline Feedback TC & o & $\mathrm{o}$ & $x$ & $\mathrm{o}$ & o \\
\hline
\end{tabular}

\subsection{Analysis on performance of primary control}

4.1.1. The initial stage of the islanded mode. When the residential community microgrid is connected to the main grid during normal operation, DERs operate according to the nominal residential electricity tariff $0.25 \$ / \mathrm{kWh}$, as shown in Table 2, where positive/ negative values represent consumption/supply quantities. When the residential community microgrid is disconnected from the main grid instantaneously, DERs are not sufficient to supply all loads. To this end, the microgrid controller immediately overwrites the guidance price at $1.2 \$ / \mathrm{kWh}$, and all DERs respond to this price. Specifically, battery systems response to the price via the incremental cost function (15), and loads adjust consumption according to formula (9), where elastic coefficients of L1 and L 2 are set as 0.4 and 0.5 . These setups make the total supply slightly higher than the total demand at the instance of islanding.

\section{Table 2. Disconnect states of DERs}

\begin{tabular}{lcc}
\hline & Grid Connected & Initial Status of Islanding \\
\hline Price $(\$ / \mathrm{kWh})$ & 0.25 & 1.2 \\
\hline L1 (p.u.) & 2.91 & 1.9 \\
\hline L2 (p.u.) & 2.19 & 1.1 \\
\hline BS1 (p.u.) & 0.35 & -1.1 \\
\hline BS2 (p.u.) & 0.18 & -1.1 \\
\hline PV (p.u.) & -0.8 & -1 \\
\hline Main grid (p.u.) & -4.83 & - \\
\hline
\end{tabular}

With the above setup, the dynamic performance of the microgrid during the islanding stage is shown in Figures 6-7. Figure 6 shows that, when the droop control begins to take actions on balancing supply and demand, both battery storages reduce their active power discharges from 1.1 p.u to around 1.05 p.u. They equally share active power supply through frequency droop control, and finally settle the system frequency to 60.01 $\mathrm{Hz} \pm 50 \mathrm{mHz}$ at $0.243 \mathrm{~s}$ [3], as shown in Figure 7. As droop settings of the two battery storage systems are the same, their dynamic behavior in Figures 6-7 are identical. It is also noteworthy that frequency of PV1 presents a larger oscillation than $\mathrm{BS} 1$ and $\mathrm{BS} 2$, because its output follows the grid frequency formed by the batteries, while the phase-lock loop (PLL) error and filter latency further enlarge the oscillation.

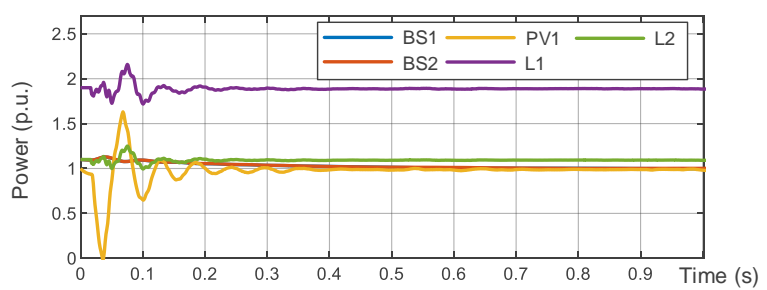

Figure 6. Dynamic power outputs during initial stage of the islanded mode

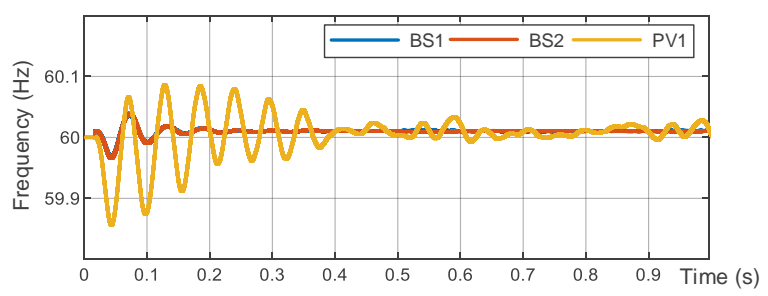

Figure 7. Dynamic frequency outputs during initial stage of the islanded mode

4.1.2. Response to disturbance. At $\mathrm{t}=2 \mathrm{~s}$, the solar irradiation is reduced, leading to $50 \%$ reduction in active power output of PV1. In order to maintain supplydemand balance, BS1 and BS2 promptly increase their active power outputs through droop control, and reduce the system frequency as shown in Figure 8. With the decrease in system frequency, frequency-responsive loads simultaneously reduce their consumptions. Therefore, the real-time supply-demand balance is ensured by increasing supply and reducing demand, and the system frequency is stabilized $0.219 \mathrm{~s}$ after disturbance, as shown in Figure 9.

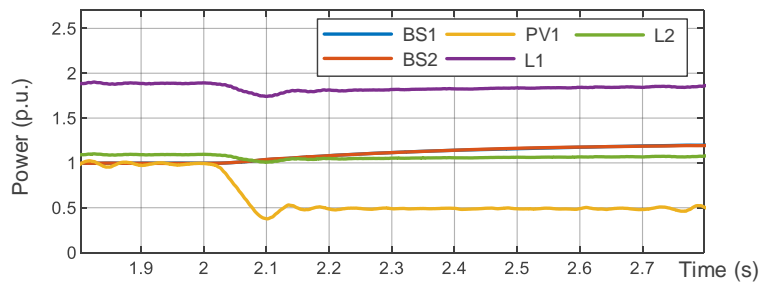

Figure 8. Power outputs against disturbance

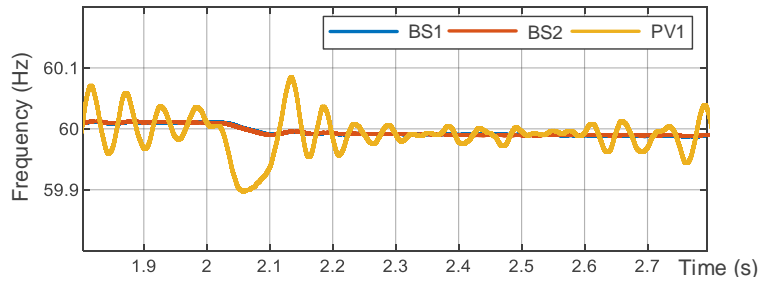

Figure 9. Frequency outputs against disturbance 


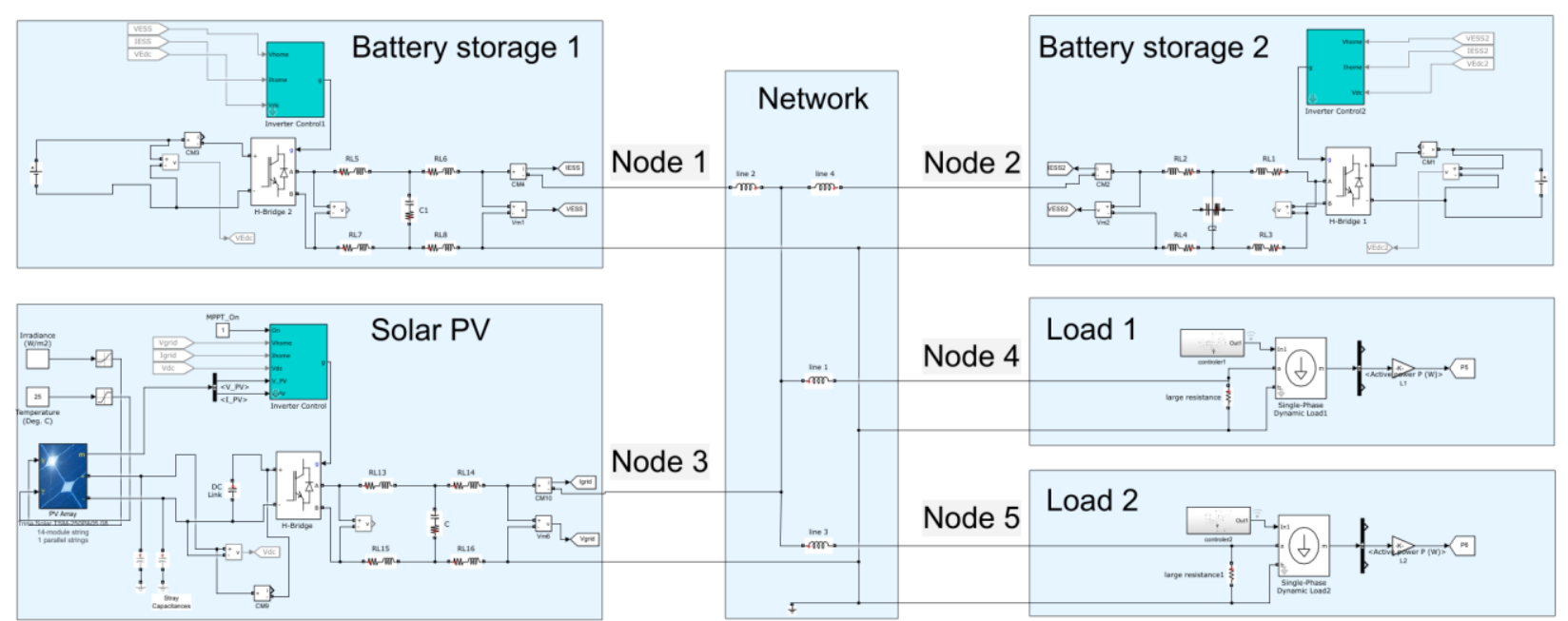

Figure 5. Topology of the residential community microgrid

\subsection{Analysis on performance of online primary- secondary cooperative feedback control}

In this subsection, we further add the secondary control loop to BS1, forming an online primarysecondary cooperative feedback control scheme. Figure 10 shows the dynamic outputs of all system assets.

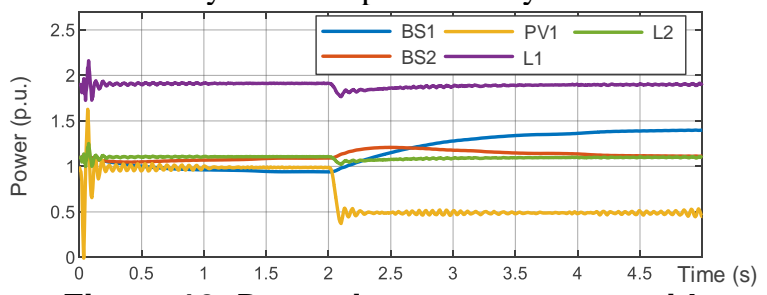

Figure 10. Dynamic power outputs with primary-secondary cooperative control

Before the disturbance occurs at $t=2 s$, the secondary control has successfully restored the system frequency to $60 \mathrm{~Hz}$, and changes on power outputs induced by the primary control has been released. That is, active power output of BS2 is recovered to its original set point 1.1 p.u., after it is initially reduced to 1.06 p.u. by the primary control to achieve instant supply-demand balance. In comparison, the accumulated frequency deviation in the secondary PI controller drives BS1 to take over the imbalance, reducing its output to 0.9 p.u.

When the disturbance occurs at $\mathrm{t}=2 \mathrm{~s}$ with $50 \%$ reduction in PV1's power output, the droop control responds promptly to increase power outputs of BS1 and BS2 for ensuring power balance. Meanwhile, the secondary control also works in real-time to gradually restore the frequency, as shown in Figure 11. To this end, BS1 actively regulates the frequency deviation by continuously increasing its output, to bring system frequency back to the nominal value. When frequency is restored to $60 \mathrm{~Hz}, \mathrm{BS} 2$ releases all its primary compensation and returns to 1.1 p.u., and BS1 stays at
1.4 p.u. due to accumulated secondary control effects.

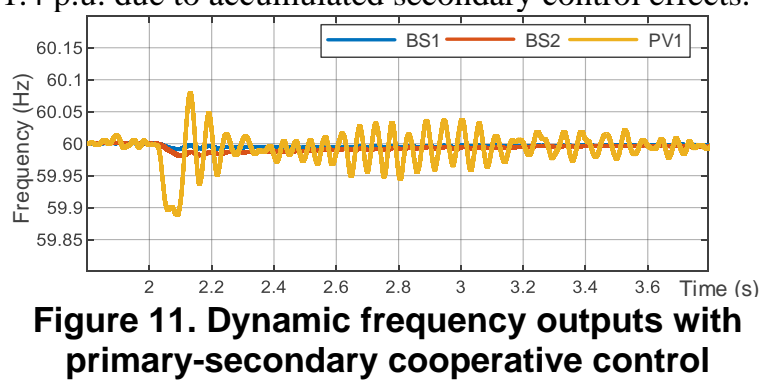

\subsection{Analysis on performance of online primary- secondary-tertiary cooperative feedback control}

Using the same system setup as the previous subsections, this subsection compares the traditional hierarchical feedforward control scheme (in Figure 1) and the proposed online cooperative feedback control scheme (in Figure 2). Specifically, control signals generated by the tertiary control layer and performance of the entire control scheme are compared and analyzed.

4.3.1. Control signals from the tertiary control layer. In the hierarchical feedforward control scheme, considering that it takes time to solve the centralized optimization problem, we activate the tertiary control at $1 \mathrm{~s}$ and $3 \mathrm{~s}$. That is, the tertiary control signals, namely the new set points of BS1, BS2, L1, and L2, are assigned at $1 \mathrm{~s}$ and again at $3 \mathrm{~s}$, as shown in Figure 12.

In comparison, the proposed online cooperative feedback control scheme generates tertiary control signals in real time, based on instantaneous difference between incremental cost of batteries and marginal price of price-sensitive loads. It is computationally inexpensive, and can provide continuous adjustment commands for batteries to pursue their economic operating points in real-time. As shown in Figure 13, the 
proposed online cooperative feedback control scheme provides effective adjustments, and guarantees that active power outputs converge to the optimal value against islanding and disturbing events (i.e., when the system converges, the control signals become zeros).

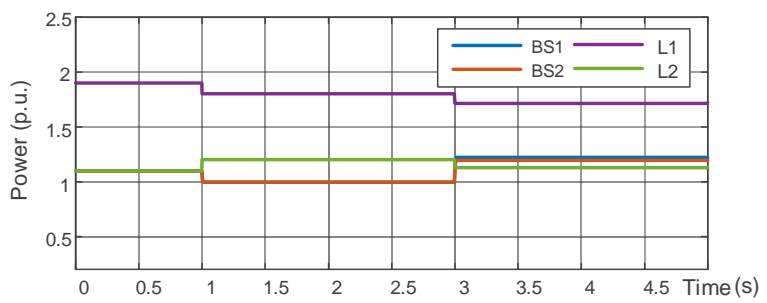

Figure 12. Tertiary control signal from the traditional hierarchical feedforward control

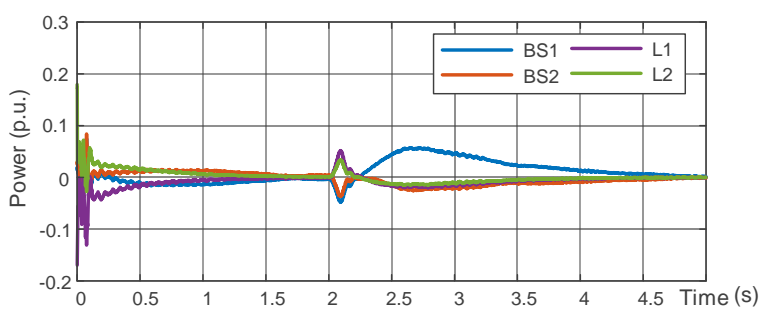

Figure 13. Tertiary control signal from the proposed online cooperative feedback control

4.3.2. Power outputs of the entire control scheme. The optimal active power outputs of batteries in an ideal situation are first calculated, which are then used as the benchmark to compare those in traditional hierarchical feedforward control scheme and the proposed online cooperative feedback control scheme. Indeed, the ideal situation calculates optimal dispatches of batteries and flexible loads against loads and PV outputs in every time instant, while neglecting the effect of power inertia on their dynamic response behavior. The optimal active power outputs of batteries derived from the ideal situation, the traditional hierarchical feedforward control scheme, and the proposed online cooperative feedback control scheme are compared in Figure 14.

Figure 14 shows that, under the traditional hierarchical feedforward control scheme, BS1 and BS2 gradually deviate from their set points when disturbance occurs at $2 \mathrm{~s}$, because of activation of the primary control. At $2.4 \mathrm{~s}$, the secondary control becomes dominating, so that BS1 continues increasing its output while BS2 begins to release its primary compensation. At 3s, when the tertiary control is activated (as shown in Figure 12) to update the new set points, outputs of BS1 and $\mathrm{BS} 2$ begin to move towards the new optimal points. This clearly shows the traditional feedforward control scheme achieves power balance, frequency recovery, and optimal operation as three staggered targets.

In comparison, the proposed online cooperative feedback control scheme can effectively coordinate all three controllers to instantly respond to fluctuations, and effectively manage supply-demand balance in real-time. Figure 14 clearly shows that power outputs of the two batteries from the proposed control scheme are closer to the ideal situation than those of the traditional control scheme. Thus, the proposed online cooperative feedback control scheme can better track the optimal operation of the system. Numerically, the cost of traditional feedforward control at $3 \mathrm{~s}$ is $4.48 \%$ higher than that of the proposed feedback control, and the total operation cost for the $5 \mathrm{~s}$ time window is $1.8 \%$ higher than the proposed cooperative feedback control scheme.

Indeed, the hierarchical feedforward control scheme could perform even worse, in terms of economic operation, if the tertiary control is activated rather less frequently. In addition, in the hierarchical feedforward control scheme, some DERs may be pushed to their critical operation status (e.g., the secondary control drives BS1 to increase its output by $40 \%$ within $1 \mathrm{~s}$ ). Thus, the system may lose stability once some DERs are operated close to or beyond their security margins.

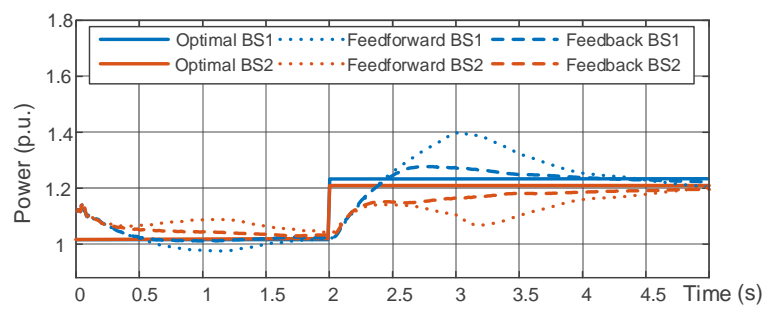

\section{Figure 14. Dynamic power outputs under different control schemes}

\section{Conclusion}

This paper discusses an online cooperative feedback control scheme for the residential community microgrid with $100 \%$ renewable and limited battery storage assets in islanded mode. Through the smallsignal analysis and the Simulink Simscape Electrical based simulation tests, the following conclusions are obtained:

1) Each of primary, secondary, and tertiary controllers is established via a feedback control loop with good real-time convergence characters; The realtime price-driven tertiary control promotes DERs and price-responsive loads better tracking their optimal operations via the incremental cost models, achieving the decentralized economic operation of residential community microgrid during islanded mode.

2) The three controllers are tightly connected in an outer feedback loop to form the proposed online cooperative feedback control, which can sustain supplydemand balance against disturbances, regulate voltage/ frequency, and track the economic operations. It presents better stability and economic performance than 
the traditional hierarchical feedforward control scheme.

In our future work, the proposed control scheme will be further tested via a hardware-in-the-loop environment to demonstrate its performance in an actual setup. In addition, we will further augment the proposed control scheme via modern predictive control and machine learning based approaches, to handle uncertainties of disturbances and complicated physical characteristics of the distribution network and DERs.

\section{Appendix}

Table 3. Control parameters

\begin{tabular}{|c|c|c|c|}
\hline \multirow{5}{*}{ 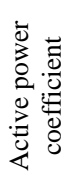 } & $K_{f, P}^{\mathrm{I}}$ & Droop control & 0.1 \\
\hline & $K_{f, P}^{\mathrm{II}}$ & Secondary control proportional term & 2 \\
\hline & $K_{f, I}^{\mathrm{II}}$ & Secondary control integral term & 5 \\
\hline & $K_{f, P}^{\text {III }}$ & ED proportional term & 2 \\
\hline & $K_{f, I}^{\mathrm{III}}$ & ED integral term & 5 \\
\hline \multirow{9}{*}{ 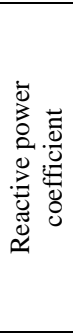 } & $K_{v, Q}^{\mathrm{I}}$ & Droop control & 0.1 \\
\hline & $K_{v, P}^{\mathrm{II}}$ & Secondary control proportional term & 2 \\
\hline & $K_{v, I}^{\mathrm{II}}$ & Secondary control integral term & 5 \\
\hline & $K_{v, P}^{\text {III }}$ & Reactive sharing proportional term & 0.4 \\
\hline & $K_{v, I}^{\mathrm{III}}$ & Reactive sharing integral term & 1 \\
\hline & $K_{P}^{\mathrm{V}}$ & Voltage loop proportional term & 5 \\
\hline & $K_{I}^{\mathrm{V}}$ & Voltage loop integral term & 12.5 \\
\hline & $K_{P}^{\mathrm{C}}$ & Current loop proportional term & 0.5 \\
\hline & $K_{I}^{\mathrm{C}}$ & Current loop integral term & 10 \\
\hline \multirow{3}{*}{ 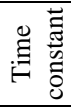 } & $\tau^{\mathrm{I}}$ & Primary filter time constant & 0.01 \\
\hline & $\tau^{\mathrm{II}}$ & Secondary filter time constant & 0.05 \\
\hline & $\tau^{\mathrm{III}}$ & Tertiary filter time constant & 0.25 \\
\hline
\end{tabular}

\section{Reference}

[1] Y. Xu, C. Liu, K.P. Schneider, F.K. Tuffner, and D.T. Ton, "Microgrids for service restoration to critical load in a resilient distribution system," IEEE Trans. Smart Grid, vol. 9, no. 1, pp. 426-437, 2016.

[2] J. Li, R. Xiong, Q. Yang, F. Liang, M. Zhang, and W. Yuan, "Design/test of a hybrid energy storage system for primary frequency control using a dynamic droop method in an isolated microgrid power system," Applied Energy, vol. 201, pp. 257-269, 2017.

[3] Q. Shafiee, J.M. Guerrero, and J.C. Vasquez, "Distributed secondary control for islanded microgridsa novel approach," IEEE Trans. Power Electronics, vol. 29, no. 2, pp. 1018-1031, 2014.

[4] H. Chvez, R. Baldick, and J. Matevosyan, "The joint adequacy of AGC and primary frequency response in single balancing authority systems," IEEE Trans. Sust. Energy, vol. 6, no. 3, pp. 959-966, 2015.

[5] F. Dörfler, J.W. Simpson-Porco, and F. Bullo, "Breaking the hierarchy: distributed control and economic optimality in microgrids," IEEE Trans. Control of Network Systems, vol. 3, no. 3, pp. 241-253, 2016.

[6] G. Zhang, J. McCalley, and Q. Wang, "An AGC dynamics-constrained economic dispatch model," IEEE Trans. Power Syst., vol. 34, no. 5, pp. 3931-3940, 2019.

[7] A. Hauswirth, A. Zanardi, S. Bolognani, F. Dörfler, and
G. Hug, "Online optimization in closed loop on the power flow manifold," 2017 IEEE Manchester PowerTech, Manchester, pp. 1-6, 2017.

[8] N. Li, C. Zhao, and L. Chen, "Connecting automatic generation control and economic dispatch from an optimization view," IEEE Trans. Control of Network Systems, vol. 3, no. 3, pp. 254-264, 2016.

[9] Y. Zhang, M. Hong, E. Dall'Anese, S.V. Dhople, and Z. $\mathrm{Xu}$, "Distributed controllers seeking AC optimal power flow solutions using ADMM," IEEE Trans. Smart Grid, vol. 9, no. 5, pp. 4525-4537, 2017.

[10] X. Wu, C. Shen, and R. Iravani, "A distributed, cooperative frequency and voltage control for microgrids," IEEE Trans. Smart Grid, vol. 9, no. 4, pp. 2764-2776, 2018.

[11] S. Wang, J. Li, and L. Wu, "An integrated distributed economic dispatch-droop control architecture for distribution system operation," 2018 IEEE Power \& Energy Society General Meeting, Portland, pp. 1-5, 2018.

[12] Y. Liu, K. Zuo, X.A. Liu, J. Liu, and J.M. Kennedy, "Dynamic pricing for decentralized energy trading in microgrids," Applied energy, vol. 228, pp. 689-699, 2018.

[13] Z. Tang, D.J. Hill, and T. Liu, "A novel consensus-based economic dispatch for microgrids," IEEE Trans. Smart Grid, vol. 9, no. 4, pp. 3920-3922, 2018.

[14] M.H. Mostafa, S.H.A. Aleem, S.G. Ali, Z.M. Ali, and A. Y. Abdelaziz, "Techno-economic assessment of energy storage systems using annualized life cycle cost of storage (LCCOS) and levelized cost of energy (LCOE) metrics," Journal of Energy Storage, 29, p. 101345, 2020.

[15] J. Wu, X. Xing, X. Liu, J. Guerrero, and Z. Chen, "Energy management strategy for grid-tied microgrids considering the energy storage efficiency," IEEE Trans. Industrial Electronics, vol. 65, no. 12, pp. 9539-9549, 2018.

[16] S. Jafarpour, V. Purba, S.V. Dhople, B. Johnson, and F. Bullo, "Small-signal stability of grid-tied inverter networks," arXiv preprint arXiv:1902.02478, 2019.

[17] J. Lai, X. Lu, X. Yu, and A. Monti, "Cluster-oriented distributed cooperative control for multiple AC microgrids," IEEE Trans. Industrial Informatics, vol. 15, no. 11, pp. 5906-5918, 2019.

[18] J. Kim, J. Guerrero, P. Rodriguez, R. Teodorescu, and K. Nam, "Mode adaptive droop control with virtual output impedances for an inverter-based flexible AC microgrid," IEEE Trans. Power Electronics, vol. 26, no. 3, pp. 689-701, 2011.

[19] J.W. Simpson-Porco, F. Dörfler, and F. Bullo, "Synchronization and power sharing for droop-controlled inverters in islanded microgrids," Automatica, vol. 49, no. 9, pp. 2603-2611, 2013.

[20] L. Lu, "Consensus-based P-f and Q-V droop control for multiple parallel-connected inverters in lossy networks," 2013 IEEE International Symposium on Industrial Electronics, Taipei, pp. 1-6, 2013.

[21] I. Duggal and B. Venkatesh, "Short-term scheduling of thermal generators and battery storage with depth of discharge-based cost model," IEEE Trans. Power Systems, vol. 30, no. 4, pp. 2110-2118, 2015.

[22] C. Ju, P. Wang, L. Goel, and Y. Xu, "A two-layer energy management system for microgrids with hybrid energy storage considering degradation costs," IEEE Trans. Smart Grid, vol. 9, no. 6, pp. 6047-6057, 2018. 